\title{
Immunogenicity and structural characterisation of an in vitro folded meningococcal siderophore receptor (FrpB, FetA)
}

\author{
Jeroen Kortekaas ${ }^{\mathrm{a}, 1}$, Shirley A. Müller ${ }^{\mathrm{b}}$, Philippe Ringler ${ }^{\mathrm{b}}$, Marco Gregorini ${ }^{\mathrm{b}}$, \\ Vincent E. Weynants ${ }^{\mathrm{c}}$, Lucy Rutten ${ }^{\mathrm{d}}$, Martine P. Bos ${ }^{\mathrm{a}, *}$, Jan Tommassen ${ }^{\mathrm{a}}$ \\ ${ }^{a}$ Department of Molecular Microbiology, Institute of Biomembranes, Utrecht University, Padualaan 8, 3584 CH Utrecht, The Netherlands \\ ${ }^{\mathrm{b}}$ Maurice E. Müller Institute for Structural Biology, Biozentrum, University of Basel, Klingelbergstrasse 70, CH-4056 Basel, Switzerland \\ ${ }^{\mathrm{c}}$ GlaxoSmithKline Biologicals, 89 Rue de l'Institut, B-1330 Rixensart, Belgium \\ ${ }^{\mathrm{d}}$ Department of Crystal and Structural Chemistry, Bijvoet Centre for Biomolecular Research, Utrecht University, \\ Padualaan 8, 3584 CH Utrecht, The Netherlands
}

Received 27 January 2006; accepted 4 April 2006

Available online 24 May 2006

\begin{abstract}
The iron-limitation-inducible protein FrpB of Neisseria meningitidis is an outer-membrane-localized siderophore receptor. Because of its abundance and its capacity to elicit bactericidal antibodies, it is considered a vaccine candidate. Bactericidal antibodies against FrpB are, however, type-specific. Hence, an FrpB-based vaccine should comprise several FrpB variants to be capable of providing broad protection. To facilitate the development of a meningococcal subunit vaccine, we have established a procedure to obtain large quantities of the protein in a native-like conformation. The protein was expressed without its signal sequence in Escherichia coli, where it accumulated in inclusion bodies. After in vitro folding, the protein was biochemically, biophysically and biologically characterised. Our results show that in vitro folded FrpB assembles into oligomers, presumably dimers, and that it induces high levels of bactericidal antibodies in laboratory animals.
\end{abstract}

(C) 2006 Elsevier SAS. All rights reserved.

Keywords: In vitro folding; Neisseria meningitidis; Outer membrane protein; Vaccine

\section{Introduction}

Neisseria meningitidis (meningococcus) is a Gram-negative bacterium that is capable of causing life-threatening

Abbreviations: CFU, colony-forming units; EDDHA, ethylenediamine di$o$-hydroxyphenylacetic acid; FrpB, Ferric-repressed protein B; FetA, Ferric enterobactin transport protein A; GMT, geometric mean titre; HBSS, Hank's balanced salt solution; HPI, hexagonally packed intermediate; IPTG, isopropyl- $\beta$-D-thiogalactopyranoside; MH, Mueller Hinton; OD, optical density; OMP, outer membrane protein; OMV, outer membrane vesicle; PMSF, phenylmethylsulfonyl fluoride; RT, room temperature; SB-12, n-dodecyl- $N, N$-dimethyl-1-ammonio-3-propanesulfonate; STEM, scanning transmission electron microscopy; TEM, transmission electron microscopy; TSB, tryptic soy broth.

* Corresponding author. Tel.: +31 30253 3017; fax: +31 302513655 .

E-mail address: m.p.bos@bio.uu.nl (M.P. Bos).

${ }^{1}$ Present address: Animal Sciences Group, Wageningen University Research, Lelystad, The Netherlands. septicaemia and meningitis. Effective vaccines based on the capsular polysaccharides of the majority of pathogenic serogroups have been developed [1]. However, a vaccine against serogroup B meningococci, which is responsible for most disease cases in North America and Europe, is not available. Development of a polysaccharide-based vaccine against serogroup B meningococci is a cumbersome task, since the type B capsule resembles human self-antigens [2]. Outer membrane vesicle (OMV) vaccines have been successfully used to combat serogroup B epidemics, but these vaccines offer little cross-protection although recent data suggest that adjustment of the procedure used to produce OMVs may solve this problem [3].

An attractive alternative for OMV vaccines would be a subunit vaccine consisting of outer membrane proteins (OMPs). Due to its abundance and its capacity to induce bactericidal antibodies, the general diffusion porin PorA is the most 
extensively studied OMP with regard to its vaccine potential. PorA induces high levels of bactericidal antibodies, which is believed to be the best correlate for protection against serogroup-B meningococci [4]. Unfortunately, however, the PorA protein shows extensive antigenic variation among strains, which complicates the development of a broad-spectrum vaccine. In the search for antigenically more conserved OMPs, proteins involved in iron acquisition are under investigation. The meningococcus is remarkably efficient in scavenging iron from its host by using a wide variety of OMP receptors, the expression of which is regulated by the availability of iron. Together, these receptors enable the meningococcus to use various human iron-loaded proteins, such as lactoferrin, transferrin and haemoglobin [5]. Furthermore, although the meningococcus does not appear to produce its own siderophores, it has the ability to use siderophores secreted by other bacteria [6]. The major iron-limitation-induced OMP of $N$. meningitidis is a $\sim 70-\mathrm{kDa}$ protein designated FrpB (Ferric-repressed protein B) [7]. The FrpB protein of Neisseria gonorrhoeae was shown to be involved in the transport of enterobactin, a siderophore produced by Escherichia coli, and it was, therefore, renamed FetA (Ferric enterobactin transport protein A). Enterobactin transport by FrpB is specific and dependent on the energy-coupling protein TonB [8]. However, as the affinity of FrpB for enterobactin is relatively low, the possibility exists that another, enterobactin-related siderophore is the actual ligand of this protein in vivo.

FrpB is considered a promising vaccine candidate to protect against meningococcal disease. Although FrpB, like PorA, shows significant antigenic variation among strains [9], its ability to induce relatively high levels of bactericidal antibodies justifies a further analysis of this protein [10,11]. Here, we describe the production of large quantities of FrpB, which were used for structural and immunological analysis. It was suggested recently that a vaccine comprising five FrpB (FetA) variants and six variants of PorA should afford protection against a large panel of hyperinvasive meningococcal isolates [12]. Together with our previously developed method to obtain large amounts of correctly folded PorA $[13,14]$, the current work enables the development of such a multivalent meningococcal subunit vaccine.

\section{Materials and methods}

\subsection{Expression and isolation of FrpB}

The FrpB protein used throughout this study was derived from $N$. meningitidis strain $\mathrm{H} 44 / 76$. The variable region of this particular FrpB was designated F3-3 [9]. The part of frpB encoding the mature protein (i.e., FrpB without its signal sequence) was PCR amplified from genomic DNA with the primer pair $5^{\prime}$-gctacatatggcagaaaataatcggaaggtc- $3^{\prime}$ and $5^{\prime}$-gctaggatccttagaacttgtagttcacgcc- $3^{\prime}$ containing NdeI and BamHI restriction sites, respectively (underlined). The resulting product was cloned into pCRII-TOPO according to the instructions of the manufacturer (Invitrogen). From there, the $\operatorname{frpB}$ fragment was obtained by digestion with $N d e I$ and
BamHI and cloned downstream of the inducible T7 promoter of plasmid pET11a (Novagen), which was digested with the same restriction enzymes. This resulted in the plasmid pET11a-frpB. E. coli strain BL21(DE3) [15], which contains the gene for T7 RNA polymerase under an isopropyl- $\beta$-D-thiogalactopyranoside (IPTG)-inducible promoter, was transformed with the plasmid. An overnight culture of the resulting strain was diluted 1:50 into fresh Luria-Bertani (LB) medium supplemented with $100 \mu \mathrm{g} / \mathrm{ml}$ ampicillin. When an optical density of 0.6 at $550 \mathrm{~nm}\left(\mathrm{OD}_{550}\right)$ was reached, protein expression was induced by the addition of $1 \mathrm{mM}$ IPTG (Sigma). After $2.5 \mathrm{~h}$ of growth, the bacteria were harvested by centrifugation and washed with $0.9 \%(\mathrm{w} /$ v) $\mathrm{NaCl}$. Cell pellets were stored at $-20{ }^{\circ} \mathrm{C}$. FrpB was present in inclusion bodies, which were isolated as described [16].

\subsection{In vitro folding of $F r p B$}

Inclusion bodies were solubilized in $20 \mathrm{mM}$ Tris- $\mathrm{HCl}$, $100 \mathrm{mM}$ glycine ( $\mathrm{pH}$ 8.0) containing either $6 \mathrm{M}$ guanidinium hydrochloride or $8 \mathrm{M}$ urea. After incubation on a turning wheel for $1.5 \mathrm{~h}$, residual membrane fragments were removed by centrifugation for $1 \mathrm{~h}$ at $356,000 \times g$. The protein concentration in the supernatant was determined with the Pierce protein assay (Pierce, Rockford, IL, USA) using BSA (Sigma) as a standard. In vitro folding of FrpB was initiated by 20 -fold dilution of fresh solubilized inclusion bodies $(50 \mathrm{mg} / \mathrm{ml})$ in $27 \mathrm{mM}$ ethanolamine ( $\mathrm{pH} 10.8$ ) containing $0.5 \%$ (w/v) n-dodecyl- $N, N$-dimethyl-1-ammonio-3-propanesulfonate (SB-12) (Fluka, Buchs, Switzerland). Prior to use, the detergent was dissolved in methanol/chloroform (1:1) and purified by passage over an $\mathrm{Al}_{2} \mathrm{O}_{3}$ column [16]. All in vitro folding experiments with FrpB were initiated at room temperature (RT), and the protein was stored at $4{ }^{\circ} \mathrm{C}$ until analysis. When appropriate, buffers were exchanged using a PD-10 desalting column (Amersham). After exchange, some aggregates were observed, which were removed by filtration through a filter with a pore size of $0.22 \mu \mathrm{m}$ (Millipore).

\subsection{SDS-PAGE and Western blotting}

Proteins were separated by standard sodium dodecyl sulphate-polyacrylamide gel electrophoresis (SDS-PAGE). To detect folded forms of FrpB, an SDS-PAGE system that we refer to as semi-native SDS-PAGE was used [17]. In this technique, to prevent denaturation of the folded proteins the slab gels are devoid of SDS, while the amount of SDS present in the electrode buffer and loading buffer depend on the SDS sensitivity of the protein of interest and is empirically determined for each protein. For FrpB, the electrode and loading buffers contained $0.01 \%$ and $0.075 \%(\mathrm{w} / \mathrm{v})$ SDS (end concentration), respectively. The samples were kept at RT before loading. To denature the protein, a loading buffer with $2 \%$ (w/v) SDS (end concentration) was used, and these samples were boiled for $2 \mathrm{~min}$ before loading. The samples were loaded on polyacrylamide gels composed of a stacking gel with 3\% (w/v) acrylamide and a 7\% (w/v) resolving gel. 
Electrophoresis was performed on ice at a constant current of $10 \mathrm{~mA}$. After electrophoresis, proteins were visualized by Coomassie brilliant blue staining.

For two-dimensional (2-D) semi-native SDS-PAGE, a lane was cut from a gel run in the first dimension. This gel slice was then rotated $90^{\circ}$ and placed on top of a new polyacrylamide gel. Electrophoresis in the second dimension was performed under the same conditions as the first dimension, after which protein spots were visualized by silver staining [18].

Western blotting was performed as described [19]. Antibodies used were a polyclonal mouse antiserum raised against in vitro folded FrpB and convalescent patients sera obtained from the Department of Medical Microbiology, Academic Medical Centre, Amsterdam, the Netherlands. Peroxidase-conjugated secondary antibodies in combination with Enhanced Chemiluminescence (Amersham) were used for detection.

\section{4. $C D$ spectroscopy}

CD spectra of FrpB in $5 \mathrm{mM}$ phosphate buffer $[0.775 \mathrm{mM}$ $\mathrm{NaH}_{2} \mathrm{PO}_{4}, 4.225 \mathrm{mM} \mathrm{Na} \mathrm{HPO}_{4}(\mathrm{pH}$ 7.6)] containing $0.3 \%$ $(\mathrm{w} / \mathrm{v}) \mathrm{SB}-12$ were recorded at RT over a wavelength range of 190-260 nm using a $0.5-\mathrm{mm}$ path cell in an Olis CD instrument (Olis Inc., USA). The monochromator was equipped with a fixed disk and gratings were used with 2400 lines/ $\mathrm{mm}$ (blaze wavelength $230 \mathrm{~nm}$ ). The FrpB concentration used for CD measurements was $0.586 \mathrm{mg} / \mathrm{ml}\left(A_{280}\right)$. The CD spectra shown are the average of ten repeated scans (step resolution $1 \mathrm{~nm}$ ) from which the corresponding buffer spectrum was subtracted. The measured $\mathrm{CD}$ signals were converted to molar ellipticity $(\theta)$. For the comparison between folded and denatured protein, a sample that had been boiled for $3 \mathrm{~min}$ in $0.1 \%$ SDS (w/v) was also analysed.

\subsection{Isolation of cell envelopes}

The PorA-deficient derivative of strain H44/76, strain CE2001 [20], was grown in TSB supplemented with $20 \mu \mathrm{g} /$ $\mathrm{ml}$ of the iron chelator ethylenediamine di-o-hydroxyphenylacetic acid (EDDHA; Sigma). Bacteria were collected by centrifugation, resuspended in $50 \mathrm{mM}$ Tris $-\mathrm{HCl}, 5 \mathrm{mM}$ EDTA ( $\mathrm{pH}$ 8.0) containing the protease-inhibitor cocktail "Complete" (Roche) and stored overnight at $-80{ }^{\circ} \mathrm{C}$. After ultrasonic disintegration, unbroken cells were removed by centrifugation $(12,000 \times g, 15 \mathrm{~min})$ and cell envelopes were collected by ultracentrifugation $(170,000 \times g, 5 \mathrm{~min})$. The resulting pellet was dissolved in $2 \mathrm{mM}$ Tris $-\mathrm{HCl}(\mathrm{pH}$ 7.6) and stored at $-80{ }^{\circ} \mathrm{C}$.

\subsection{Trypsin digestion}

A sample of in vitro folded FrpB $[0.5 \mathrm{mg} / \mathrm{ml}$ in $50 \mathrm{mM}$ Hepes ( $\mathrm{pH} 7.5), 0.3 \%(\mathrm{w} / \mathrm{v})$ SB-12] was divided between two vials. One sample was denatured by boiling in $0.1 \%$ (w/ v) SDS for $5 \mathrm{~min}$, the other was left untreated. Both samples were subsequently treated with trypsin (Sigma) for 15 min at RT, after which $1 \mathrm{mM}$ phenylmethylsulfonyl fluoride
(PMSF; Sigma) was added and the samples were analysed by SDS-PAGE. For N-terminal sequencing, the tryptic fragments were blotted from the gels onto polyvinylidene difluoride membranes (Millipore). The blots were stained with Coomassie blue G-250, and appropriate bands were excised. Samples were subjected to five steps of Edman degradation at the Protein Sequencing Facility, Utrecht University, Utrecht, the Netherlands.

Trypsin sensitivity of FrpB present in cell envelopes was assessed by incubating cell envelopes with $10 \mathrm{mg} / \mathrm{ml}$ trypsin for $30 \mathrm{~min}$ at RT. Then, $1 \mathrm{mM}$ PMSF was added and the samples were analysed by SDS-PAGE, followed by Western blotting.

\subsection{Scanning transmission electron microscopy (STEM)}

The FrpB sample $[10 \mu \mathrm{g} / \mathrm{ml}$ in phosphate buffer ( $\mathrm{pH} 7.6)$, $0.3 \%(\mathrm{w} / \mathrm{v}) \mathrm{SB}-12]$ was diluted $50 \times$ with buffer containing detergent. Immediately afterwards, 5- $\mu$ l aliquots were adsorbed for $60 \mathrm{~s}$ to glow-discharged STEM films (thin carbon films that span a thick fenestrated carbon layer covering 200mesh/inch, gold-plated copper grids). The grids were then blotted, washed on 7 drops of quartz double-distilled water, and freeze-dried at $-80{ }^{\circ} \mathrm{C}$ and $5 \times 10^{-8}$ Torr overnight in the microscope. The hexagonally packed intermediate (HPI) surface layer of the bacterium Deinococcus radiodurans (kindly supplied by Dr. Harald Engelhardt, Max-Planck-Institute for Biochemistry, Martinsried, Germany) served as mass standard. This sample was similarly adsorbed to separate microscopy grids, washed on 4 droplets of quartz double-distilled water, and air-dried.

A Vacuum Generators STEM HB-5 interfaced to a modular computer system (Tietz Video and Image Processing Systems $\mathrm{GmbH}$, Gauting, Germany) was employed. Series of $512 \times$ 512-pixel, dark-field images were recorded from the unstained samples at an accelerating voltage of $80 \mathrm{kV}$ and a nominal magnification of $200,000 \times$. The recording dose ranged from 448 to 855 electrons $/ \mathrm{nm}^{2}$. Further, repetitive low dose scans were made of some grid regions to assess beam-induced mass-loss as outlined in [21]. The digital images were evaluated using the program package IMPSYS [21]. Accordingly, the projections were selected in circular boxes, and the total scattering of each region was calculated. The background scattering of the carbon support film was then subtracted and the mass was calculated. The results were scaled according to the mass measured for HPI and corrected for the beam-induced mass-loss, which varied between $8.1 \%$ and $15.4 \%$ depending on the dose employed. The mass values were then displayed in histograms and described by Gauss curves.

\subsection{Transmission electron microscopy (TEM)}

For TEM analysis, 5- $\mu \mathrm{l}$ aliquots of FrpB [20 $\mu \mathrm{g} / \mathrm{ml}$ in phosphate buffer ( $\mathrm{pH} 7.6), 0.3 \%(\mathrm{w} / \mathrm{v}) \mathrm{SB}-12]$ were adsorbed for $60 \mathrm{~s}$ to a glow-discharged, carbon-coated TEM microscopy grid. The grid was then blotted, washed on 7 droplets of quartz 
double-distilled water and negatively stained on 2 droplets of $2 \%(\mathrm{w} / \mathrm{v})$ uranyl acetate $(\mathrm{pH} 4.5)$. Bright-field images were recorded on photographic film using a Hitachi 7000 electron microscope at an accelerating voltage of $100 \mathrm{kV}$ and a magnification of $80,000 \times$. A Leafscan scanner was employed to digitise two of the negatives at a sampling step size of $0.5 \mathrm{~nm} /$ pixel, yielding 24 subimages $(512 \times 512$ pixels $)$. Projections (centred in $32 \times 32$-pixel regions) were automatically selected from these by cross-correlation with a centred, 20-fold symmetrised circular reference, using the SEMPER image-processing package (Synoptics Ltd., Cambridge, UK). Reference-free alignment and classification of the selected particles were done with the SPIDER image processing software [22].

\subsection{Immunisation of mice}

A group of 10 OF-1 mice (Charles River, Lyon, female, 6-8 weeks of age) received three injections with in vitro folded FrpB in $50 \mathrm{mM}$ Hepes (pH 7.5), 0.3\% (w/v) SB-12 by intramuscular route on days 0,21 and 28 . With each injection, $5 \mu \mathrm{g}$ of antigen formulated in $\mathrm{AlPO}_{4}$ plus monophosphoryl lipid A was administered. Ten control mice received adjuvant only. Blood samples were collected 14 days after the third injection.

\subsection{ELISA}

ELISA plates were coated with $100 \mu$ of folded FrpB $(5 \mu \mathrm{g} / \mathrm{ml})$ in PBS. After overnight incubation at $4{ }^{\circ} \mathrm{C}$, the plates were washed three times with $150 \mathrm{mM} \mathrm{NaCl}, 0.05 \%$ $(\mathrm{v} / \mathrm{v})$ Tween-20. Non-specific protein-binding sites were blocked with $200 \mu \mathrm{l}$ PBS, $1 \%$ (w/v) BSA for $30 \mathrm{~min}$ at RT under agitation. After washing with $150 \mathrm{mM} \mathrm{NaCl}, 0.05 \%$ (v/v) Tween-20, serum was added to the wells and serially diluted (twofold dilution steps) in PBS, 0.2\% (w/v) BSA, $0.05 \%(\mathrm{v} / \mathrm{v})$ Tween-20. After $30 \mathrm{~min}$, the plates were washed and subsequently incubated first with goat anti-mouse $\mathrm{IgG}$ antibodies conjugated to biotin (Prosan, 1:2000) and then with streptavidin-peroxidase (Amersham, 1:3000) in the same buffer. Incubations were performed for $30 \mathrm{~min}$ at $37^{\circ} \mathrm{C}$ under shaking. Plates were washed and incubated in the dark with $0.1 \mathrm{M}$ citrate buffer ( $\mathrm{pH} 4.5$ ), $0.4 \%$ (w/v) $o$-phenylenediamine (Sigma), $2 \mathrm{mM} \mathrm{H}_{2} \mathrm{O}_{2}$. The reaction was stopped by the addition of $1 \mathrm{M} \mathrm{HCl}$ and the absorbance was read at $490 \mathrm{~nm}$.

\subsection{Serum bactericidal assay}

$N$. meningitidis strain $\mathrm{H} 44 / 76$ and its frpB mutant derivative CE1431 [7] were grown overnight on Mueller Hinton (MH) agar (Difco), $1 \%$ (v/v) Polyvitex (Biomérieux), 1\% (v/v) horse serum (Sigma) at $37{ }^{\circ} \mathrm{C}$ in a $5 \% \mathrm{CO}_{2}$ atmosphere. The bacteria were inoculated in tryptic soy broth (TSB) (Beckton Dickinson) with $50 \mu \mathrm{M}$ of the iron chelator desferrioxamine mesylate (Sigma) and grown in shaking flasks for $3 \mathrm{~h}$ at $37{ }^{\circ} \mathrm{C}$ until an $\mathrm{OD}_{470}$ of 0.5 was reached. The complement in pooled serum was heat inactivated for $40 \mathrm{~min}$ at $56{ }^{\circ} \mathrm{C}$. The serum was subsequently diluted 1:100 in Hank's balanced salt solution (HBSS, Gibco), $0.3 \%$ (w/v) BSA and then serially diluted (two-fold dilution steps, eight dilutions) in a volume of $50 \mu \mathrm{l}$ in round-bottom microtitre plates. Bacteria were diluted in HBSS, $0.3 \%(\mathrm{w} / \mathrm{v}) \mathrm{BSA}$ to yield 13,000 colony-forming units (CFU) per ml. Afterwards, 37.5- $\mu$ l aliquots of this dilution were added to the serum dilutions and the microtitre plates were incubated for $15 \mathrm{~min}$ at $37^{\circ} \mathrm{C}$ under shaking. Subsequently, $12.5 \mu \mathrm{l}$ of baby-rabbit complement (Pelfreez) or, as a control for toxicity of the sera, heat-inactivated complement $\left(56{ }^{\circ} \mathrm{C}\right.$ for $45 \mathrm{~min}$ ) was added to the wells. After $1 \mathrm{~h}$ of incubation at $37^{\circ} \mathrm{C}$ under shaking, the microtitre plates were placed on ice to stop killing. A $20-\mu l$ aliquot was removed from each well and spotted on MH agar plates containing $1 \%(\mathrm{v} / \mathrm{v})$ Polyvitex, $1 \%(\mathrm{v} / \mathrm{v})$ horse serum. Plates were tilted to allow the drop to "run" down the plate. After overnight incubation at $37{ }^{\circ} \mathrm{C}$ in a $5 \% \mathrm{CO}_{2}$ atmosphere, the CFUs were counted. Bactericidal titres were defined as the reciprocal of the highest serum dilution yielding $>50 \%$ killing. A PorA-specific antiserum from mice immunised with PorA-containing OMVs was used for reference in the bactericidal assays.

\subsection{Immunofluorescence microscopy}

Bacteria were grown in candle jars at $37{ }^{\circ} \mathrm{C}$ in a humid atmosphere on GC (Oxoid) plates supplemented with Vitox (Oxoid) and with $20 \mu \mathrm{g} / \mathrm{ml}$ EDDHA to impose iron limitation. The next day, the bacteria were suspended in HBSS, $0.5 \%$ (w/v) BSA (washing buffer) to an $\mathrm{OD}_{550}$ of 1. Bacteria from $300 \mu \mathrm{l}$ of this suspension were collected by centrifugation and resuspended in $300 \mu \mathrm{l}$ washing buffer containing serum (1:100). After incubation for $1 \mathrm{~h}$ and washing twice, the bacteria were resuspended in $300 \mu \mathrm{l}$ of washing buffer containing the secondary antibody goat anti-mouse IgG conjugated with Alexa-green (Molecular Probes, OR, USA) (1:400). After incubation for $1 \mathrm{~h}$ and washing, the bacteria were collected and resuspended in $300 \mu \mathrm{l}$ HBSS containing $2 \%(\mathrm{v} / \mathrm{v})$ formaldehyde, immobilized on polylysine-coated coverslips and analysed by microscopy.

\section{Results}

\subsection{Expression and purification of FrpB}

The $\operatorname{frpB}$ gene without the signal-sequence-encoding region was cloned behind the T7 promoter on pET11a, and the construct was introduced into E. coli strain BL21(DE3), which contains the gene for T7 polymerase under an IPTG-inducible promoter. Upon induction, expression of the recombinant $f r p B$ gene resulted in large amounts of FrpB (Fig. 1A, lane 2). The majority of FrpB was present in inclusion bodies, which were isolated and solubilized in a chaotropic agent. The protein yield was estimated as $500 \mathrm{mg} / \mathrm{l}$ of bacterial culture. SDSPAGE analysis demonstrated the high purity of the solubilized protein (Fig. 1A, lane 3), rendering additional purification steps unnecessary. Although $\operatorname{FrpB}$ has a calculated $M_{\mathrm{r}}$ of 


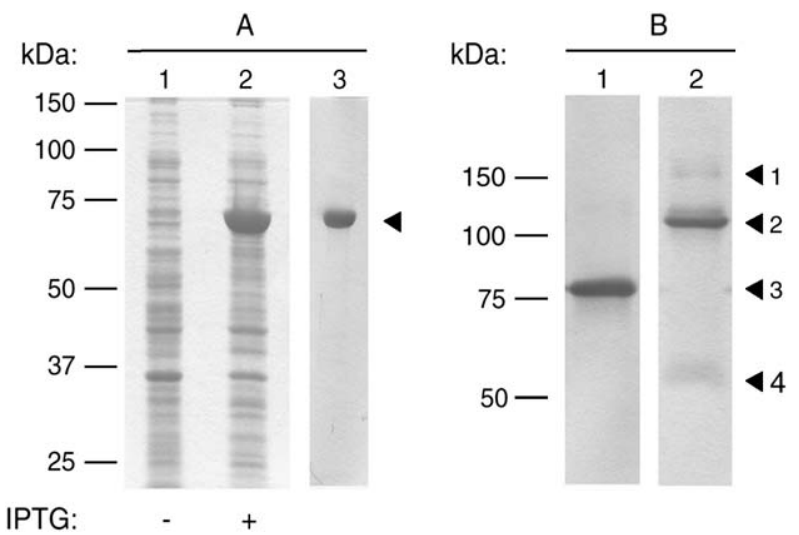

Fig. 1. Expression, purification and in vitro folding of recombinant FrpB. (A) Whole cell lysates of $E$. coli BL21(DE3) containing pET11a-frpB incubated without (lane 1) or with (lane 2) IPTG. Lane 3 shows the protein pattern of the inclusion bodies solubilized in $8 \mathrm{M}$ urea. The arrowhead indicates FrpB. Samples were incubated at $100{ }^{\circ} \mathrm{C}$ in loading buffer containing $2 \%$ SDS prior to electrophoresis. (B) In vitro folded FrpB was heat denatured by boiling for 2 min in loading buffer containing 2\% SDS (lane 1) or incubated at RT in loading buffer containing $0.075 \%$ SDS (lane 2) and analysed by semi-native SDS-PAGE. The positions of different heat-modifiable forms (arrowheads 1,2 and 4) and the denatured form (arrowhead 3) of FrpB are indicated. The positions of the molecular-weight standard proteins are indicated on the left of both panels.

77,348 based on its amino acid sequence, the protein migrated as a $70,000 M_{\mathrm{r}}$ protein.

\subsection{In vitro folding of FrpB}

Correctly folded $\beta$-barrel OMPs exhibit deviant electrophoretic mobility on semi-native SDS-PAGE. Due to their compact conformation the folded monomeric proteins usually migrate more quickly on gels than the heat-denatured form, whereas oligomers migrate more slowly [13]. This so-called heat modifiability of OMPs can be used to monitor their folding in vitro $[13,16]$.

To identify appropriate folding conditions, stock solutions of FrpB in $6 \mathrm{M}$ guanidinium hydrochloride were diluted in a range of buffers, containing different detergents and salts and having various $\mathrm{pHs}$, to a series of final protein and denaturant concentrations. Initially, analysis of FrpB using routine semi-native SDS-PAGE did not reveal any differences in electrophoretic mobility. However, since native FrpB from cell envelopes appeared to be rather sensitive to SDS (data not shown), the amount of SDS normally applied in semi-native SDS-PAGE was lowered. Eventually, folded forms of FrpB were visualized and optimal folding conditions could be determined.

After dilution of guanidinium hydrochloride-solubilized FrpB in $27 \mathrm{mM}$ ethanolamine ( $\mathrm{pH} 10.8$ ), 0.5\% (w/v) SB-12, semi-native SDS-PAGE revealed a major band migrating with an $M_{\mathrm{r}}$ of 110,000 (Fig. 1B, lane 2). Furthermore, two minor bands with $M_{\mathrm{r}}$ of 155,000 and 55,000 were detected. The $55,000-M_{\mathrm{r}}$ band probably corresponds to a folded monomeric form of FrpB, and the $110,000-M_{\mathrm{r}}$ band to a dimer of two folded monomers, whereas the $155,000-M_{\mathrm{r}}$ band suggests that some trimers might also be present. Although in vitro folding was always very efficient, the ratio of these folded forms varied between experiments.

Only a single band, representing the unfolded protein, was detected after heat treatment (Fig. 1B, lane 1). Strikingly, the unfolded protein migrated more slowly on semi-native SDS-PAGE gels than on normal SDS-PAGE gels (Fig. 1A, lane 3). This discrepancy is most likely the result of the limiting amounts of SDS present in the electrode buffer during semi-native SDS-PAGE, since analysis of the same samples by regular SDS-PAGE revealed the expected $70,000-M_{\mathrm{r}}$ band (data not shown).

\subsection{Semi-native 2-D electrophoresis}

The detection of both monomers and oligomers of FrpB in semi-native SDS-PAGE suggested that these forms might be in equilibrium. This possibility was tested by semi-native 2-D SDS-PAGE, in which the electrophoretic conditions applied were identical in both directions. In these experiments, folded monomers detected after migration in the first dimension revealed oligomers after migration in the second dimension and vice versa, consistent with an equilibrium state (Fig. 2).

\section{4. $C D$ analysis}

In their native conformation, OMPs are barrels with a high $\beta$-sheet content. The secondary structure of in vitro folded FrpB was evaluated by CD measurements. The CD spectrum had a minimum at $217 \mathrm{~nm}$, consistent with the presence of $\beta$-sheet secondary structure (Fig. 3, solid line). The spectrum

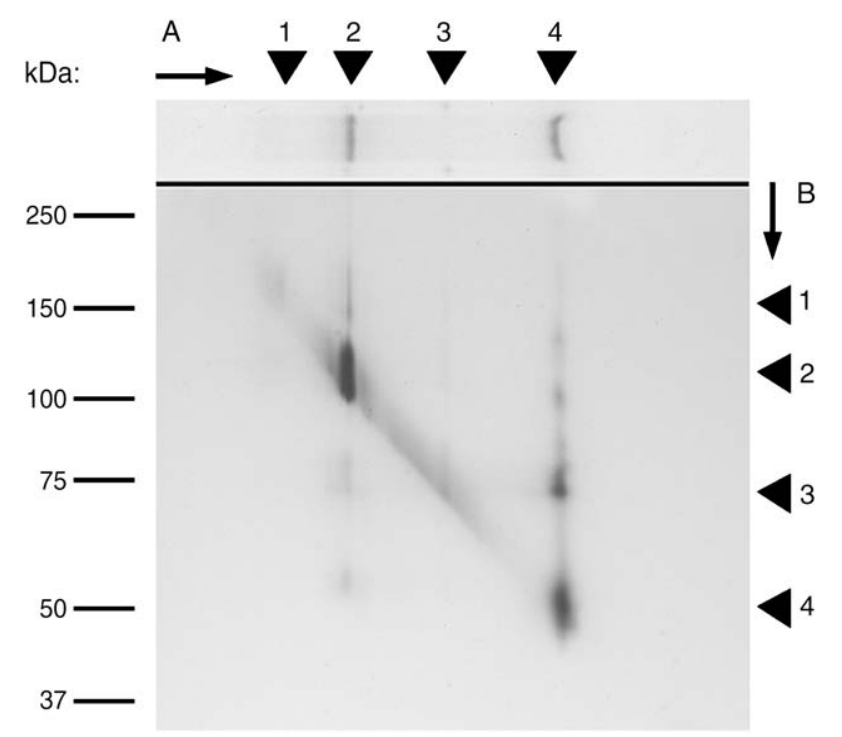

Fig. 2. Semi-native 2-D SDS-PAGE analysis. In vitro folded FrpB was analysed in the first (arrow A) and the second (arrow B) dimension by semi-native SDS-PAGE. The positions of different heat-modifiable forms (arrowheads 1, 2 and 4 ) and the denatured form (arrowhead 3) of FrpB are indicated. Proteins were visualized by Coomassie staining (first dimension) or silver staining (second dimension). The positions of the molecular-weight standard proteins are indicated on the left. 


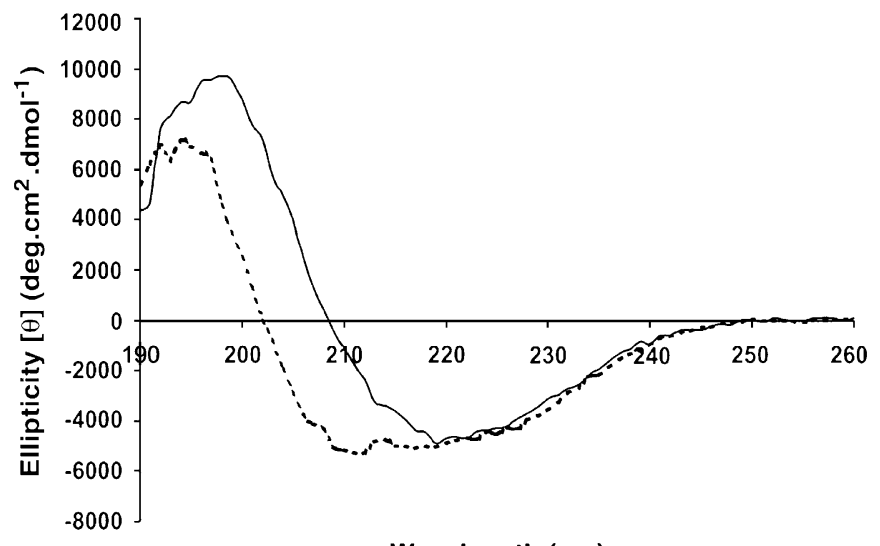

Wavelength $(\mathrm{nm})$

Fig. 3. CD spectra of in vitro folded FrpB (solid line) and FrpB denatured by boiling in $0.1 \%$ SDS (dotted line).

of a sample that had been denatured by boiling in the presence of $0.1 \%(w / v)$ SDS (Fig. 3, dotted line) showed much more $\alpha$-helical content, consistent with results reported for other SDS-denatured OMPs (e.g. [16]).

\subsection{Trypsin digestion}

Trypsin-digestion experiments were performed to confirm that FrpB was folded in a native-like conformation. Folded proteins are usually more resistant to proteases than the unfolded protein or folding intermediates. Denatured FrpB was completely degraded with $10 \mu \mathrm{g} / \mathrm{ml}$ trypsin (Fig. 4A). In contrast, when folded FrpB was treated with the same amount of trypsin, two distinct tryptic fragments were detected one of which migrated slightly more quickly than the untreated protein (Fig. 4B, T1). N-terminal sequencing of this fragment revealed the amino acid sequence VVLDT, indicating that trypsin cleaved the protein after Lys-6. N-terminal sequencing of the second tryptic fragment, with an $M_{\mathrm{r}}$ of $\sim 50,000$ (Fig. 4B, T2), revealed the amino acid sequence INIDR, which

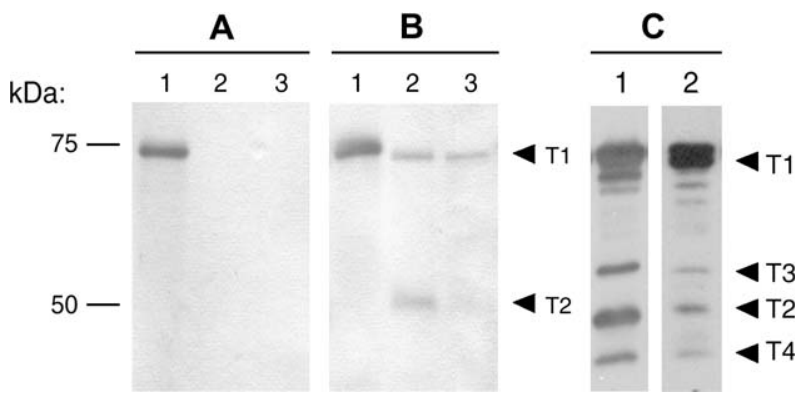

Fig. 4. Trypsin digestion of FrpB. (A) Denatured and (B) in vitro folded FrpB were treated with 0 (lane 1), 10 (lane 2) or 100 (lane 3 ) $\mu \mathrm{g} / \mathrm{ml}$ trypsin and the tryptic fragments were separated by SDS-PAGE and stained with Coomassie brilliant blue. T1 and T2 indicate the major tryptic fragments of FrpB. (C) Tryptic fragments of FrpB present after incubation of in vitro folded FrpB with $10 \mu \mathrm{g} / \mathrm{ml}$ trypsin (lane 1) and cell envelopes with $10 \mathrm{mg} / \mathrm{ml}$ trypsin (lane 2) were detected on Western blots. Arrowheads indicate tryptic fragments. The positions of the molecular-weight standard proteins are indicated on the left. resulted from cleavage after Arg-249. According to a proposed topology model of FrpB (unpublished), both of these trypsinsensitive sites correspond to exposed regions in the native structure, i.e. the periplasm-exposed $\mathrm{N}$ terminus and the cellsurface-exposed loop L3, respectively. Strikingly, the large tryptic fragment $\mathrm{T} 1$ was unaffected when the trypsin concentration was increased to $100 \mu \mathrm{g} / \mathrm{ml}$, whereas the $50,000-M_{\mathrm{r}}$ fragment was no longer detected (Fig. 4B, lane 3). Apparently, a proportion of the in vitro folded protein is susceptible to trypsin cleavage in loop L3 and, subsequently, to further degradation, whereas the other proportion is not. This finding suggests that at least two structurally distinct forms of FrpB were formed during the in vitro folding procedure.

Besides T1 and T2, two additional tryptic fragments of FrpB were detected on Western blots (T3 and T4, Fig. 4C, lane 1). Similar fragments obtained after digestion of in vitro folded FrpB were also obtained upon trypsin treatment of cell envelopes (Fig. 4C, lane 2). These results confirm that FrpB adopted a native-like structure upon in vitro folding.

\subsection{Electron microscopic evaluation of the FrpB conformation}

To study the conformation of in vitro folded FrpB in further detail, the protein was diluted $50 \times$, adsorbed to thin carbon films and imaged by STEM for mass measurement (see Section 2). In spite of this large dilution, some particles were still too close to neighbours prohibiting evaluation. Nevertheless, over 3800 projections were selected from the images, sorted according to their dimensions into a main class and a minor class, and analysed. The mass histogram from the main class $(n=3326)$ displayed a single major peak with a distinct high-mass sidearm (Fig. 5A). Over $86 \%$ of the particles had a mass of $149,000 \pm 53,000(n \sim 2894)$. The siderophore receptor FhuA of $E$. coli, which may have a similar structure to FrpB, is reported to associate with 185 molecules of the detergent dodecyl maltoside [23]. Assuming that a similar number of detergent molecules associates with FrpB (mass predicted from the sequence $77,348 \mathrm{Da}$ ), the contribution of SB-12 (monomer mass 335.5 Da) would be around 62,000 Da, resulting in a theoretical total mass of 139,348 Da for the FrpBdetergent complex. Thus, the mass calculated from the images strongly implies the presence of a large population of FrpB monomers. The high-mass asymmetry of the histogram (Fig. 5A) reflects the presence of FrpB dimers $(n \sim 433)$, as confirmed by the Gaussian fitted to this region that falls at a mass of $263,000 \pm 53,000$. The calculated mass indicates that there is less detergent associated per monomer in the dimer, which can be explained by the absence of detergent at the site where the two monomers are in contact. The minor second class of particles $(n=505)$ was comprised of particles that were visibly larger than those in the main class and had masses in the range of dimers and above (data not shown). Accordingly, all together, $75 \%$ of all the particles selected were monomers.

An overview of the FrpB sample after negative staining with $2 \%$ uranyl acetate is shown in Fig. 5B; various 
A
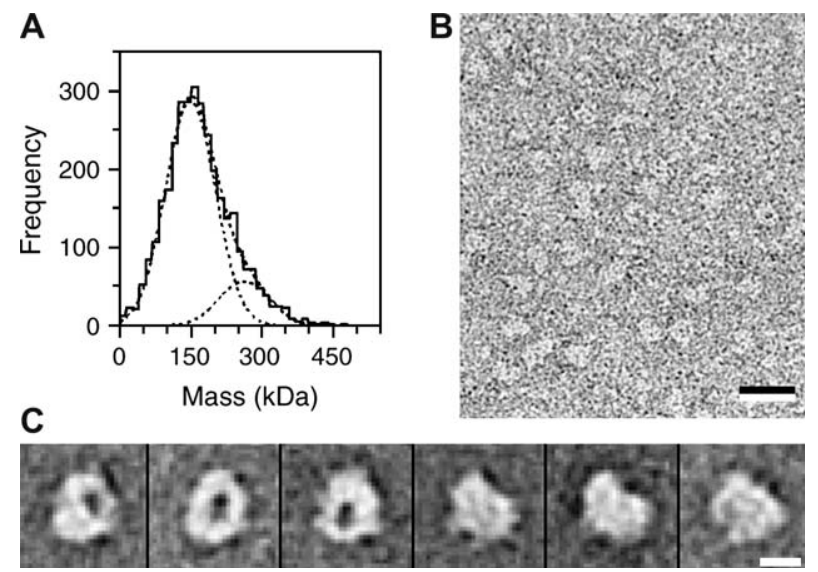

Fig. 5. Analysis of in vitro folded FrpB by electron microscopy. (A) STEM mass analysis of detergent solubilized, in vitro folded FrpB. The histogram of mass values can be described by two Gaussian curves falling at $149,000 \pm 53,000 \mathrm{Da}(n \sim 2894$; standard error, $\mathrm{SE}= \pm 1000$; overall uncertainty \pm 8000 , considering the SE and the $5 \%$ calibration uncertainty of the instrument $)$ and $263,000 \pm 53,000 \mathrm{Da} \quad(n \sim 433 ; \quad \mathrm{SE}= \pm 3000 ; \quad$ overall uncertainty $\pm 13,000$ ) respectively. (B) Overview image of the FrpB sample recorded by TEM after negative staining with $2 \%$ uranyl acetate. (C) Average projections showing tilted top-views (first three panels) and various side-views of the negatively stained, detergent solubilized FrpB monomer. The top-views indicate a stain-filled cavity implying the presence of a pore. The scale bar is $20 \mathrm{~nm}$ in (B) and $5 \mathrm{~nm}$ in (C).

orientations of the protein can be distinguished. A total of 1791 projections were automatically selected from the images and manually refined; projections too close to neighbours or clearly arising from larger particles were removed. The remaining 880 projections were then subjected to referencefree alignment and classification. Fig. 5C shows class averages of tilted top-views and side-views of the FrpB monomer. The side-view averages have diameters of $\sim 6 \mathrm{~nm}$ and $\sim 8 \mathrm{~nm}$, which is in the range expected from the crystal structures of the E. coli siderophore receptors FepA [24] and FhuA [25]. Some of the tilted top-view projections show signs of a pore (Fig. 5C).

\subsection{Immune response against in vitro folded FrpB}

Mice were immunised with in vitro folded FrpB to evaluate whether antibodies can be raised that recognize FrpB expressed in vivo at the bacterial cell surface. The resultant sera were pooled and evaluated in ELISAs using in vitro folded FrpB as the coating antigen. The geometric mean titre (GMT) of 4837 indicated good responses. Pooled control sera obtained from mice immunised with adjuvant only showed a titre of $<50$.

The specificity of the sera was further tested by immunoblotting of bacterial lysates obtained from control and iron-deprived cultures (Fig. 6A). Pooled control sera did not react with any protein in the lysates (Fig. 6B), whereas the pooled anti-FrpB serum clearly reacted with the $70 \mathrm{kDa}$, iron-limitation-inducible FrpB band (Fig. 6C). Furthermore, immunofluorescence microscopy showed that the antibodies recognized FrpB on the cell surface of living wild-type meningococci
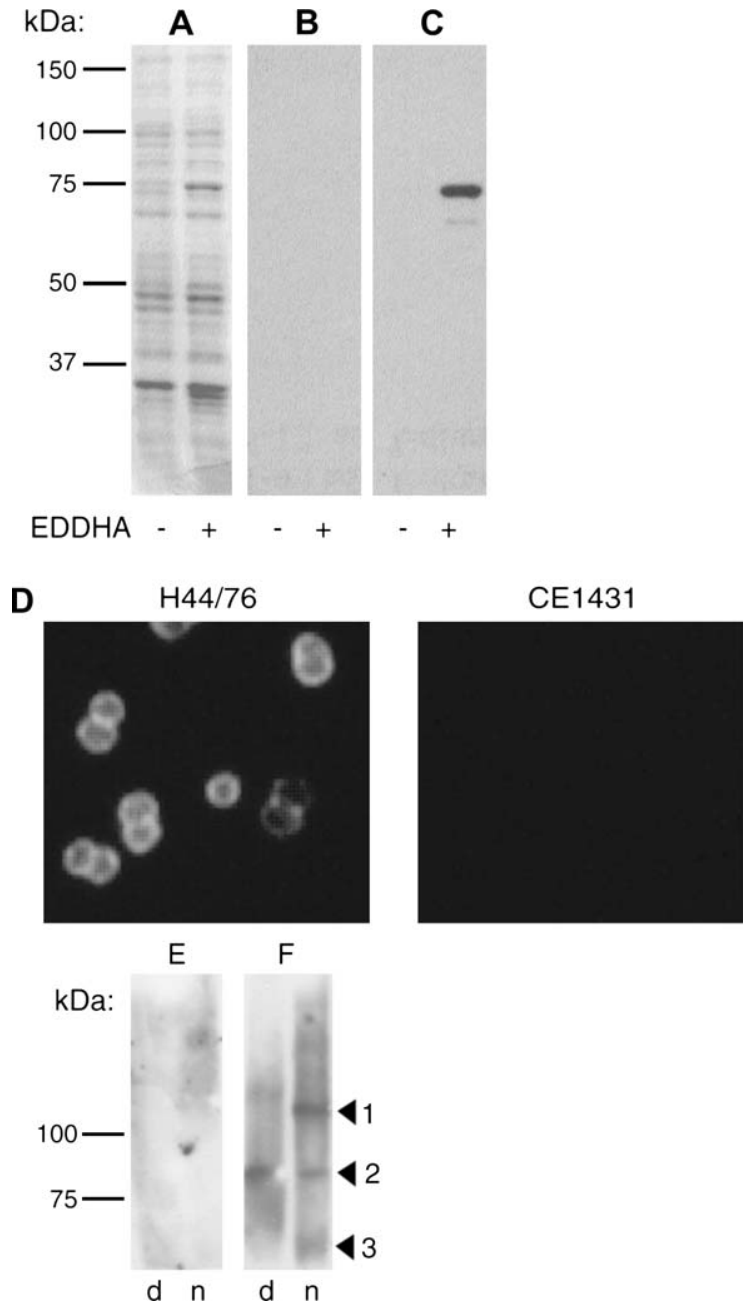

Fig. 6. Immunogenicity of in vitro folded FrpB. Proteins in lysates of porAmutant strain CE2001, grown in the presence or absence of EDDHA, were separated by SDS-PAGE and stained with Coomassie Blue (A), or blotted and probed with control serum (B) or anti-FrpB serum (C). (D) Immunofluorescence micrographs of $N$. meningitidis strain $\mathrm{H} 44 / 76$ and its frpB mutant derivative CE1431 labelled with antiserum raised against in vitro folded FrpB. $(\mathrm{E}, \mathrm{F})$ In vitro folded FrpB was denatured (d) or left native (n) and subjected to semi-native SDS-PAGE, blotted and probed with 1:500 dilutions of convalescent patient sera. (E) negative serum of a patient infected with a serogroup B strain; (F) positive serum of a patient infected with a serogroup $C$ strain. The positions of different heat-modifiable forms (arrowheads 1 and 3) and the denatured form (arrowhead 2) of FrpB are indicated. The FrpB variable region antigen types of the infecting strains are unknown.

grown under iron-limitation, whereas an $\operatorname{frpB}$ mutant was not labelled (Fig. 6D). Next, bactericidal activity of individual sera was tested. Bactericidal titres of the anti-FrpB sera against wild-type bacteria ranged from 177 to 4686 (GMT 912). These sera did not kill frpB-mutant bacteria. The control sera were not bactericidal for either wild-type or $\operatorname{frp} B$-mutant bacteria. The lack of killing of the $f r p B$ mutant was due to the absence of FrpB and not to a difference in sensitivity to complement, since the wild-type and $\operatorname{frpB} B$-mutant strain were equally sensitive to PorA-specific antiserum (GMT 1348 and 1885 , respectively). Together, these results show that native epitopes are present after folding $\mathrm{FrpB}$ in vitro and that 
bactericidal antibodies can be raised with the in vitro folded protein.

FrpB antibodies can be found in sera from patients convalescent for meningococcal disease [26]. To test whether in vitro folded FrpB can be used to identify such antibodies, we tested reactivity of 10 different convalescent patient sera with refolded FrpB on Western blots. Two out of 10 sera tested positive: a negative and positive example are shown in Fig. $6 \mathrm{E}$ and $\mathrm{F}$, respectively.

\section{Discussion}

FrpB of $N$. meningitidis is considered an important vaccine candidate. Here, we have developed a procedure to obtain large quantities of FrpB in its native conformation for both immunological and structural analysis. Semi-native SDS-PAGE indicated that the predominant form of in vitro folded FrpB is a dimer, which is in equilibrium with folded monomers and, possibly, higher oligomeric forms. In agreement with this observation, SDS-PAGE analysis of FrpB from meningococcal cell envelopes has shown that the protein is present in an oligomeric, probably dimeric, complex in the outer membrane [27]. Accurate comparison of the oligomeric structure of FrpB from meningococcal outer membranes and in vitro folded FrpB is difficult, however, since in vivo the complex interacts with the RmpM protein, which remains associated to FrpB during semi-native SDS-PAGE [27]. Although oligomers of FrpB were also detected by analysis of cell envelopes from an rmpM mutant [27], the fuzziness of these bands prohibited the accurate determination of the $M_{\mathrm{r}}$. Furthermore, these oligomers might contain associated lipopolysaccharide, which has been shown to increase the observed $M_{\mathrm{r}}$ of OMPs [28,29].

Support for the assumption that FrpB folded into a nativelike structure was obtained in trypsin sensitivity assays. Four tryptic fragments were detected on Western blots that migrated at the same position as the bands obtained upon digestion of native FrpB in cell envelopes. Two of the four bands were present in sufficient amounts for $\mathrm{N}$-terminal sequencing. One fragment resulted from cleavage after Lys-6 near the $\mathrm{N}$ terminus, the other from cleavage after Arg-249, which is located in a predicted surface-exposed loop. Lys-6 is located close to the TonB box, which supposedly interacts with the energy-coupling protein TonB. Interestingly, the E. coli siderophore receptor FhuA is cleaved by trypsin at Lys-5, which is also in close proximity to the TonB box [30]. As it is conceivable that both trypsin-sensitive sites of in vitro folded FrpB are exposed in the native structure, these results strongly indicate that the protein folded correctly during the in vitro folding procedure. Strikingly, treatment of FrpB with an increased amount of trypsin only yielded the fragment resulting from cleavage at Lys-6. This result indicates that at least two structurally different FrpB molecules coexist. Since FrpB is also present as a dimer in the preparations, it is tempting to speculate that this dimer is asymmetric.

Although mass analysis by STEM revealed the presence of dimers, it indicated that most of the FrpB protein analysed was monomeric. Thus, the monomer/dimer ratios observed from semi-native SDS-PAGE and STEM do not fully correspond. This is understandable because not every particle imaged by STEM could be measured (see Section 3). Further, the dilution required may have shifted the monomer-dimer equilibrium towards the monomer.

The current work demonstrates the ability of FrpB to assemble into homo-oligomeric dimers. Interestingly, the crystal structures of other TonB-dependent receptors, such as FhuA [25,31] and FepA [24], revealed monomeric proteins. Analytical ultracentrifugation also indicated that purified FhuA is monomeric [32]. However, in the latter study oligomers of FhuA were detected by analytical ultracentrifugation after cross-linking, and a positive co-operativity was observed in siderophore-binding experiments, which is also consistent with an oligomeric state of the receptor. Furthermore, FhuA dimers have been visualized in 2-D crystals by electron microscopy [33]. It is therefore tempting to speculate that the TonB-dependent receptors in general form dimers, which, however, readily dissociate into monomers when extracted from the membrane with detergents.

The dimensions of FrpB observed by TEM correspond to those expected from the crystal structures of FhuA [25,31] and FepA [24]. Some of the projections showed signs of a pore. These projections probably represent FrpB molecules in the up orientation, since the periplasmic side of the $\beta$-barrels of the TonB-dependent receptors is closed by a plug formed by an N-terminal domain of these proteins. Since milligram quantities of folded FrpB can now readily be obtained, future experiments will focus on elucidating its three-dimensional structure.

The in vitro folded FrpB protein was capable of inducing high levels of antibodies that recognized FrpB in the outer membrane and were bactericidal, showing that native epitopes are formed during the in vitro folding procedure. It was recently reported that a vaccine based on six PorA and five FrpB (FetA) variants should be able to provide protection against a large range of hyperinvasive meningococcal strains [12]. The current work, together with previously developed methods to fold the PorA protein in vitro [13,14], thus enables the development of a protein-based meningococcal subunit vaccine.

\section{Acknowledgements}

We thank Remco Nagel and Suzanne Hermeling for assistance in the trypsin sensitivity assays and CD analysis, respectively. Furthermore, we thank Mark Doeven and Bert Poolman (Department of Biochemistry, University of Groningen), Andreas Engel (Maurice E. Müller Institute, Biozentrum, University of Basel) and Karel Planken (Van't Hoff Laboratory for Physical and Colloid Chemistry, Utrecht University) for helpful discussions. This work was supported by the Netherlands Research Council for Chemical Sciences (CW) with financial aid from the Netherlands Technology Foundation (STW), the European Community (grant HPRN-CT2000-00075), the Maurice E. Müller Foundation with financial 
aid from the Swiss National Foundation grants NF 31-59 415.99 and SNF 501221 and the National Centre of Competence in Research Structural Biology.

\section{References}

[1] M. Mitka, New vaccine should ease meningitis fears, JAMA 293 (2005) 1433-1434.

[2] J. Finne, M. Leinonen, P.H. Mäkela, Antigenic similarities between brain components and bacteria causing meningitis. Implications for vaccine development and pathogenesis, Lancet 2 (1983) 355-357.

[3] G. Ferrari, I. Garguso, J. Abu-Dobie, F. Doro, A.R. Taddei, A. Biolchi, B. Brunelli, M.M. Giuliani, M. Pizza, N. Norais, G. Grandi, Outer membrane vesicles from group B Neisseria meningitidis $\Delta$ gna33 mutant: Proteomic and immunological comparison with detergent-derived outer membrane vesicles, Proteomics 6 (2006) 1856-1866.

[4] E.R. van der Voort, P. van der Ley, J. van der Biezen, S. George, O. Tunnela, H. van Dijken, B. Kuipers, J. Poolman, Specificity of human bactericidal antibodies against PorA P1.7, 16 induced with a hexavalent meningococcal outer membrane vesicle vaccine, Infect. Immun. 64 (1996) 2745-2751.

[5] D. Perkins-Balding, M. Ratliff-Griffin, I. Stojiljkovic, Iron transport systems in Neisseria meningitidis, Microbiol. Mol. Biol. Rev. 68 (2004) $154-171$.

[6] J.M. Rutz, T. Abdullah, S.P. Singh, V.I. Kalve, P.E. Klebba, Evolution of the ferric enterobactin receptor in Gram-negative bacteria, J. Bacteriol. 173 (1991) 5964-5974.

[7] A. Pettersson, A. Maas, D. van Wassenaar, P. van der Ley, J. Tommassen, Molecular characterization of FrpB, the 70-kilodalton iron-regulated outer membrane protein of Neisseria meningitidis, Infect. Immun. 63 (1995) 4181-4184.

[8] S.D.B. Carson, P.E. Klebba, S.M.C. Newton, P.F. Sparling, Ferric enterobactin binding and utilization by Neisseria gonorrhoeae, J. Bacteriol. 181 (1999) 2895-2901.

[9] E.A. Thompson, I.M. Feavers, M.C.J. Maiden, Antigenic diversity of meningococcal enterobactin receptor FetA, a vaccine component, Microbiology 149 (2003) 1849-1858.

[10] D.A.A. Ala'Aldeen, H.A. Davies, S.P. Borriello, Vaccine potential of meningococcal FrpB: studies on surface exposure and functional attributes of common epitopes, Vaccine 12 (1994) 535-541.

[11] A. Pettersson, B. Kuipers, M. Pelzer, E. Verhagen, R.H. Tiesjema, J. Tommassen, J.T. Poolman, Monoclonal antibodies against the 70-kilodalton iron-regulated protein of Neisseria meningitidis are bactericidal and strain specific, Infect. Immun. 58 (1990) 3036-3041.

[12] R. Urwin, J.E. Russell, E.A.L. Thompson, E.C. Holmes, I.M. Feavers, M.C.J. Maiden, Distribution of surface protein variants among hyperinvasive meningococci: implications for vaccine design, Infect. Immun. 72 (2004) 5955-5962.

[13] C. Jansen, A. Wiese, L. Reubsaet, N. Dekker, H. de Cock, U. Seydel, J. Tommassen, Biochemical and biophysical characterization of in vitro folded outer membrane porin PorA of Neisseria meningitidis, Biochim. Biophys. Acta 1464 (2000) 284-298.

[14] C. Jansen, B. Kuipers, J. van der Biezen, H. de Cock, P. van der Ley, J. Tommassen, Immunogenicity of in vitro folded outer membrane protein PorA of Neisseria meningitidis, FEMS Immunol. Med. Microbiol. 27 (2000) 227-233.

[15] F.W. Studier, B.A. Moffatt, Use of bacteriophage T7 RNA polymerase to direct selective high-level expression of cloned genes, J. Mol. Biol. 189 (1986) 113-130.
[16] N. Dekker, K. Merck, J. Tommassen, H.M. Verheij, In vitro folding of Escherichia coli outer-membrane phospholipase A, Eur. J. Biochem. 232 (1995) 214-219.

[17] R. Voulhoux, M.P. Bos, J. Geurtsen, M. Mols, J. Tommassen, Role of a highly conserved bacterial protein in outer membrane protein assembly, Science 299 (2003) 262-265.

[18] H. Blum, H. Beier, H.J. Gross, Improved silver staining of plant proteins, RNA and DNA in polyacrylamide gels, Electrophoresis 8 (1987) 93-99.

[19] P. van Ulsen, L. van Alphen, J. ten Hove, F. Fransen, P. van der Ley, J. Tommassen, A neisserial autotransporter NalP modulating the processing of other autotransporters, Mol. Microbiol. 50 (2003) 1017-1030.

[20] J. Tommassen, P. Vermeij, M. Struyvé, R. Benz, J.T. Poolman, Isolation of Neisseria meningitidis mutants deficient in class 1 (porA) and class 3 ( porB) outer membrane proteins, Infect. Immun. 58 (1990) 1355-1359.

[21] S.A. Müller, K.N. Goldie, R. Bürki, R. Häring, A. Engel, Factors influencing the precision of quantitative scanning transmission electron microscopy, Ultramicroscopy 46 (1992) 317-334.

[22] J. Frank, M. Radermacher, P. Penczek, J. Zhu, Y. Li, M. Ladjadj, A. Leith, SPIDER and WEB: processing and visualization of images in 3D electron microscopy and related fields, J. Struct. Biol. 116 (1996) 190-199.

[23] P. Boulanger, M. le Maire, M. Bonhivers, S. Dubois, M. Desmadril, L. Letellier, Purification and structural and functional characterization of FhuA, a transporter of the Escherichia coli outer membrane, Biochemistry 35 (1996) 14216-14224.

[24] S.K. Buchanan, B.S. Smith, L. Venkatramani, D. Xia, L. Esser, M. Palnitkar, R. Chakraborty, D. van der Helm, J. Deisenhofer, Crystal structure of the outer membrane active transporter FepA form Escherichia coli, Nat. Struct. Biol. 6 (1999) 56-63.

[25] A.D. Ferguson, E. Hofman, J.W. Coulton, K. Diederichs, W. Welte, Siderophore-mediated iron transport: crystal structure of FhuA with bound lipopolysaccharide, Science 282 (1998) 2215-2220.

[26] E. Wedege, E.A. Høiby, E. Rosenqvist, G. Bjune, Immune response against major outer membrane antigens of Neisseria meningitidis in vaccinees and controls who contracted meningococcal disease during the Norwegian serogroup B protection trial, Infect. Immun. 66 (1998) 3223-3231.

[27] T. Prinz, J. Tommassen, Association of iron-regulated outer membrane proteins of Neisseria meningitidis with the RmpM (class 4) protein, FEMS Microbiol. Lett. 183 (2000) 49-53.

[28] M.P. Bos, B. Tefsen, J. Geurtsen, J. Tommassen, Identification of an outer membrane protein required for the transport of lipopolysaccharide to the bacterial cell surface, Proc. Natl. Acad. Sci. U.S.A. 101 (2004) 9417-9422.

[29] C. Jansen, M. Heutink, J. Tommassen, H. de Cock, The assembly pathway of outer membrane protein PhoE of Escherichia coli, Eur. J. Biochem. 267 (2000) 3792-3800.

[30] G.S. Moeck, P. Tawa, H. Xiang, A.A. Ismail, J.L. Turnbull, J.W. Coulton, Ligand-induced conformational change in the ferrichrome-iron receptor of Escherichia coli K-12, Mol. Microbiol. 22 (1996) 459-471.

[31] K.P. Locher, B. Rees, R. Koebnik, A. Mitscher, L. Mouinier, J.P. Rosenbusch, D. Moras, Transmembrane signaling across the ligand-gated FhuA receptor: crystal structures of free and ferrichromebound states reveal allosteric changes, Cell 95 (1998) 771-778.

[32] K.P. Locher, J.P. Rosenbusch, Oligomeric states and siderophore binding of the ligand-gated FhuA protein that forms channels across Escherichia coli outer membranes, Eur. J. Biochem. 247 (1997) 770-775.

[33] O. Lambert, G.S. Moeck, D. Levy, L. Plançon, L. Letellier, J.L. Rigaud, An 8 - $\AA$ projected structure of FhuA, a "ligand-gated" channel of the Escherichia coli outer membrane, J. Struct. Biol. 126 (1999) 145-155. 